\title{
Long-term color variations of the peculiar X-ray binary V Sagittae
}

\author{
V. Simon ${ }^{1}$, S. Shugarov ${ }^{2}$, and V. I. Marsakova ${ }^{3}$ \\ 1 Astronomical Institute, Academy of Sciences, 25165 Ondřejov, Czech Republic \\ 2 Sternberg Astronomical Institute, Moscow State University, Universitetsky Prospect 13, 119899 Moscow, Russia \\ 3 Department of Astronomy, Odessa State University, T.G. Shevchenko park, Odessa, 65014, Ukraine \\ Received 29 February 2000 / Accepted 26 October 2000

\begin{abstract}
We present an analysis of the color variations of the X-ray binary V Sge during high, medium and low state of its activity over the years 1995-1997, using $U B V$ data. We resolved the track of V Sge in the color diagrams $(B-V$ and $U-B$ versus $\operatorname{mag}(V))$ during transitions between the states and show that the color variations on the orbital time scale are significantly smaller than the changes caused by the long-term activity. The mean $B-V$ decreases by 0.15 mag during the upper part of the transition (brightness higher than $11.5 \operatorname{mag}(V)$ ) from the low to the high state but stays almost constant below this level. On the contrary, $U-B$ does not change significantly during the whole transition. Comparison of our data with those of Herbig et al. (1965) shows that although the character of the long-term activity in V Sge changed significantly during the last decades (Šimon \& Mattei 1999) the colors and their variations with the brightness level remained similar.
\end{abstract}

Key words. stars: activity - binaries: general - circumstellar matter - novae, cataclysmic variables stars: individual: V Sge

\section{Introduction}

V Sge is a peculiar and very active binary $\left(P_{\text {orb }}=0.514 \mathrm{~d}\right)$ (Herbig et al. 1965, hereafter HPSP) which has been detected over a broad spectral range, from X-ray (Eracleous et al. 1991; Hoard et al. 1996; Greiner \& van Teeseling 1998) and UV (Koch et al. 1986) to radio (Lockley et al. 1997). The observations imply a very high mass transfer or outflow rate of the order of $10^{-6} M_{\odot} \mathrm{yr}^{-1}$ (Lockley et al. 1997).

The recent findings strongly support the model, originally suggested by Williams et al. (1986), in which the white dwarf (WD) primary, surrounded by a disk, accretes matter from a more massive, but less luminous companion. The evidence is based mainly on the $\mathrm{X}$-ray variations (Greiner \& van Teeseling 1998), bearing the resemblance to the behaviour of the supersoft X-ray binaries in the Magellanic Clouds, and on the high-excitation emission lines and a very high ratio $E W(\mathrm{He}$ II 4686$) / E W(\mathrm{H} \beta)$ which need a powerful source of soft X-rays (Patterson et al. 1998). An additional argument is the character of the long-term activity (Šimon \& Mattei 1999 - hereafter Paper I) which bears many common links to the VY Scltype and some supersoft X-ray binaries. Paper I brought

Send offprint requests to: V. Šimon, e-mail: simon@asu.cas.cz

* Table 2 is only available in electronic form at the CDS via anonymous ftp to cdsarc.u-strasbg.fr (130.79.128.5) or via

http://cdsweb.u-strasbg.fr/cgi-bin/qcat?J/A+A/366/100 the arguments that the interplay between the high/low state transition and variations of the dimension of the primary can hardly be explained if the primary is a lobe filling star, suggested earlier by HPSP. A discussion of the observed contradictions of the respective models and a way to reconcile them was also presented. V Sge is a promising candidate for a super-soft X-ray binary (SSXB) (Steiner \& Diaz 1998; see van den Heuvel et al. 1992 for the basic model of SSXB).

V Sge displays a very strong photometric activity (e.g. HPSP, Robertson et al. 1997, Paper I). High and low states, alternating with a typical amplitude of 1.5 to 2 mag on the time scale of tens to hundreds of days are the most common feature of the present light curve. However, the character of the activity evolves - relatively isolated outbursts occurred in 1930's and even in 1960's V Sge spent more time in low state of brightness. The intervals of the alternating high and low states are sometimes interrupted by segments of suppressed brightness variations (called flat segments in Paper I), in which the light curve stays relatively stable with just minor variations by about $0.5 \mathrm{mag}$.

In this paper we analyze the color variations during various states of activity and transitions between them, using $U B V$ photometric data.

\section{The observations}

The photoelectric observations in Johnson $U B V$ system were secured in three seasons, separated by the long gaps 


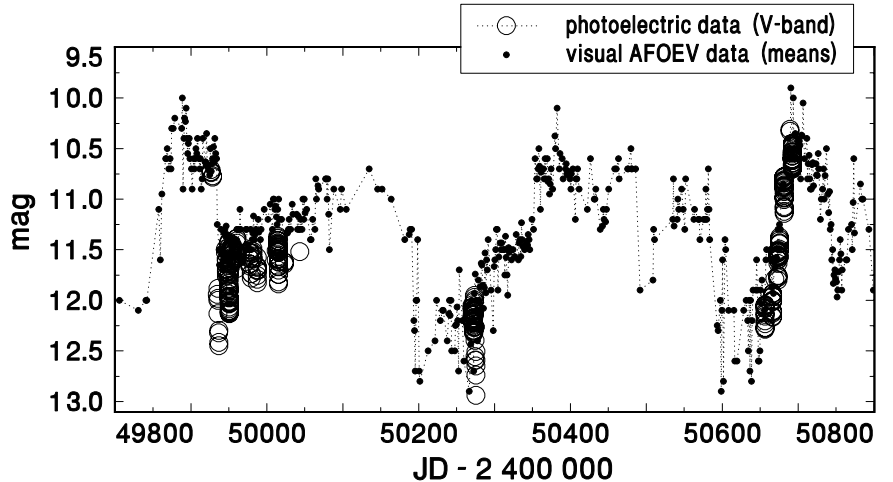

Fig. 1. The light curve illustrating the character of activity in V Sge over the years 1995-1997. The photoelectric $V$-band data of all orbital phases are plotted as the large empty circles, the superposed one-day means of the out-eclipse (phase 0.1-0.9) AFOEV visual observations are marked as dots

(July-November 1995; July 1996; August 1997). The measurements in the first two seasons were obtained with the 600/7500 mm Cassegrain telescope at Crimea Observatory while the 700/10 500 mm Cassegrain telescope at Moscow Observatory of Sternberg Astronomical Institute was used for observations of the third run. The observations were transformed into standard Johnson system. Part of the 1995 data has been already published in Lockley et al. (1999). Tables 1 and 2 present the journal of the observations and sample of the data. The latter table in its entirety is available electronically from CDS.

$\mathrm{V}$ Sge is known to have an optical companion (14.4 $\operatorname{mag}(V), 9.7 \operatorname{arcsec}$ distant in position angle $267^{\circ}$ ) (HPSP). Diameter of the diaphragm was 14 arcsec in most cases, a few measurements were obtained with 20 arcsec diameter. The contribution of this companion was therefore at least largely minimized.

The star USNO-A2.0 1050-17330568 ( $V=10.70, B-$ $V=0.29, U-B=0.32)$ was used as the comparison star while USNO-A2.0 1050-17224337 $(V=9.49, B-V=$ $0.15, U-B=0.32)$ served as the check star. Series of simultaneous $U B V$ measurements, covering up to several hours, were secured in most nights. In addition, dense series in a single filter aiming at detection of the rapid changes were obtained in some nights.

\section{Analysis of the data}

In order to illustrate which kind of activity our $U B V$ observations represent, the visual light curve is superposed in Fig. 1. It consists of one-day means of the out-eclipse observations (phase 0.1-0.9). These visual data come from the database of Association Française des Observateurs d'Étoiles Variables (AFOEV), operated at CDS, France.

The role of the long-term and the orbital color changes can be assessed from series of the densely spaced $U B V$ observations which cover segments of the orbital light curve in various levels of the out-eclipse brightness. The examples are displayed in Figs. 2a-d. The scales of $B-V$ and $U-B$ diagrams are identical for all plots and the mean
Table 1. Journal of the observations. The middle column gives the number of observations obtained in $V, B$ and $U$-filters, respectively. The epochs and orbital phases were calculated according to the quadratic ephemeris of Smak (1995). Typical exposure time was $10 \mathrm{~s}$

\begin{tabular}{|c|c|c|}
\hline Date & No. $(V, B, U)$ & Epoch + phase \\
\hline 27/28 Jul. 95 & $3,3,0$ & $23408.215-23408.231$ \\
\hline 29/30 Jul. 95 & $2,2,2$ & $23412.436-23412.445$ \\
\hline 4/5 Aug. 95 & $1,1,1$ & 23424.037 \\
\hline 5/6 Aug. 95 & $4,4,0$ & $23426.097-23426.167$ \\
\hline 6/7 Aug. 95 & $4,4,4$ & $23427.950-23427.974$ \\
\hline 15/16 Aug. 95 & $2,2,2$ & $23445.601-23445.613$ \\
\hline 16/17 Aug. 95 & $15,14,14$ & $23447.175-23447.556$ \\
\hline 19/20 Aug. 95 & $13,13,13$ & $23452.947-23453.276$ \\
\hline 20/21 Aug. 95 & $29,29,27$ & $23454.933-23455.253$ \\
\hline 21/22 Aug. 95 & $14,14,11$ & $23457.134-23457.308$ \\
\hline 22/23 Aug. 95 & $8,8,8$ & $23458.840-23458.884$ \\
\hline 24/25 Aug. 95 & $15,15,15$ & $23462.679-23462.882$ \\
\hline $30 / 31$ Aug. 95 & $9,9,3$ & $23474.316-23474.514$ \\
\hline 16/17 Sep. 95 & $8,8,8$ & $23507.391-23507.503$ \\
\hline 18/19 Sep. 95 & $3,3,3$ & $23511.572-23511.603$ \\
\hline 20/21 Sep. 95 & $7,7,6$ & $23515.263-23515.417$ \\
\hline 26/27 Sep. 95 & $8,8,8$ & $23526.862-23526.923$ \\
\hline $21 / 22$ Oct. 95 & $2,2,0$ & $23575.502-23575.517$ \\
\hline 23/24 Oct. 95 & $14,14,14$ & $23579.144-23579.322$ \\
\hline $24 / 25$ Oct. 95 & $25,25,25$ & $23581.080-23581.220$ \\
\hline 1/2 Nov. 95 & $3,3,3$ & $23596.643-23596.663$ \\
\hline $21 / 22$ Nov. 95 & $1,1,0$ & 23635.546 \\
\hline 5/6 Jul. 96 & $15,15,15$ & $24077.514-24077.651$ \\
\hline 6/7 Jul. 96 & $9,9,9$ & $24079.429-24079.608$ \\
\hline 7/8 Jul. 96 & $12,12,12$ & $24081.399-24081.535$ \\
\hline 8/9 Jul. 96 & $8,8,8$ & $24083.209-24083.306$ \\
\hline 9/10 Jul. 96 & $5,5,4$ & $24085.084-24085.141$ \\
\hline 10/11 Jul. 96 & $5,5,4$ & $24087.061-24087.124$ \\
\hline 26/27 Jul. 97 & $15,15,15$ & $24828.149-24828.266$ \\
\hline 5/6 Aug. 97 & $15,15,15$ & $24847.447-24847.711$ \\
\hline 8/9 Aug. 97 & $1,1,1$ & 24853.322 \\
\hline 12/13 Aug. 97 & $17,17,17$ & $24861.148-24861.360$ \\
\hline 14/15 Aug. 97 & $8,8,8$ & $24865.063-24865.114$ \\
\hline 20/21 Aug. 97 & $25,24,11$ & $24876.657-24876.963$ \\
\hline 28/29 Aug. 97 & $2,2,2$ & $24892.221-24892.237$ \\
\hline 31 Aug./1 Sep. 97 & $39,38,36$ & $24897.883-24898.269$ \\
\hline
\end{tabular}

value of the color index for the given run is marked. The typical $1 \sigma$ errors of $V B U, B-V$ and $U-B$ are marked. These errors were determined under the assumption of the constant color indices over the whole night series, so they should be taken rather as upper limits. The quadratic ephemeris of Smak (1995) was used for calculation of the orbital phases:

$$
\begin{aligned}
T(\text { prim.min. })= & 2437889.9157+0.51419706 E \\
& -1.2310^{-10} E^{2} .
\end{aligned}
$$

Variations of the color index $B-V$ with the orbital phase for various levels of out-eclipse brightness in the $V$-band is shown in Fig. 3. The division of brightness reflects the levels in which V Sge spends most time and corresponds to the states of activity, discussed in Sect. 4 . Smoothing by 

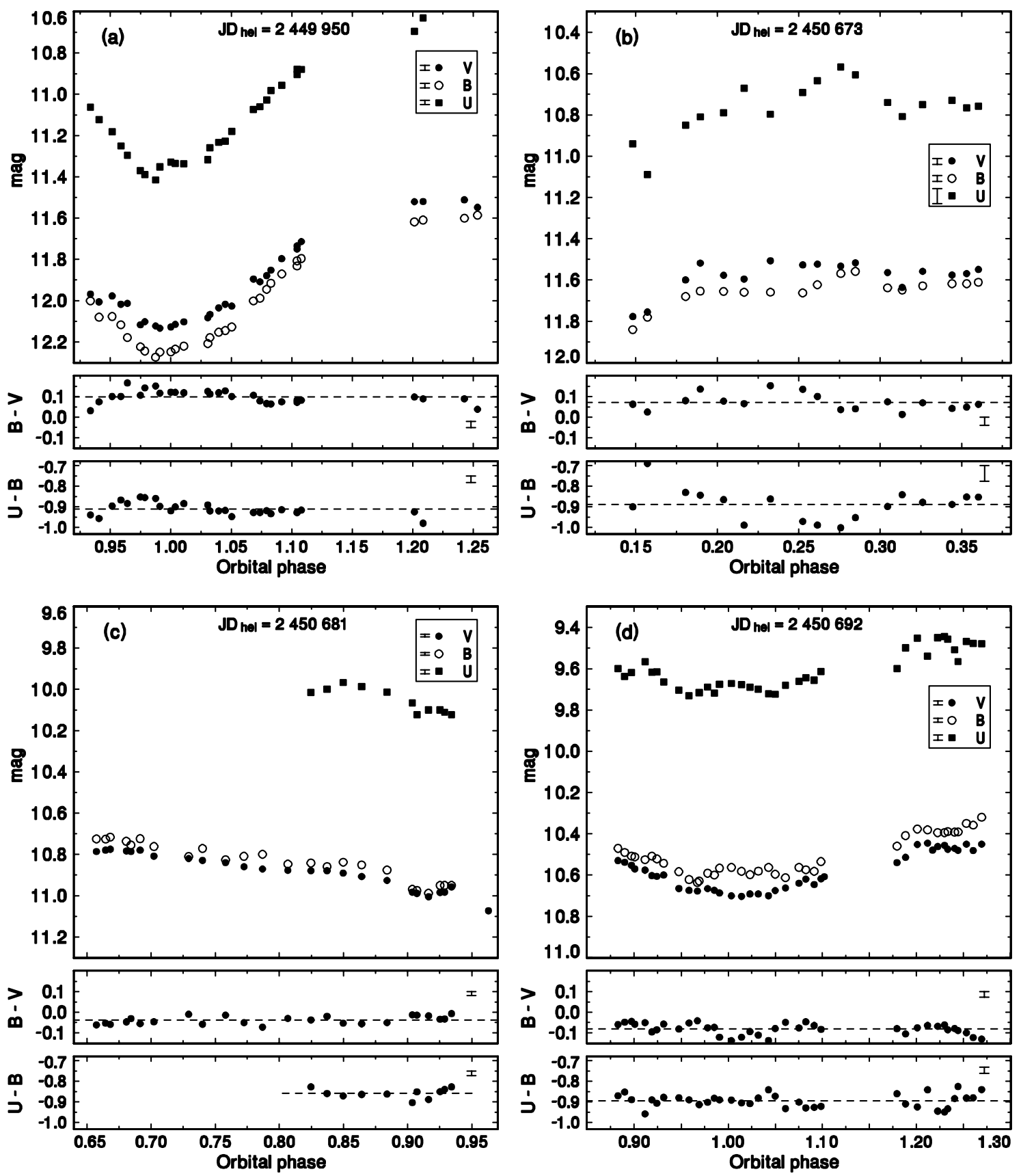

Fig. 2. Orbital modulation in $U B V$ passbands and the corresponding changes of the color indices. a) 20 August 1995 (orbital epochs $E=23454$ and 23455$)$; b) 12 August $1997(E=24861)$; c) 20 August $1997(E=24876)$; d) 31 August 1997 ( $E=24897$ and 24898). The dashed lines denote the mean value of the color index for the given run. The error bars in each panel denote the typical $1 \sigma$ errors of $V B U, B-V$ and $U-B$. The observations are plotted in real time but the horizontal axis is expressed in units of the orbital phase instead of Julian Date. The quadratic ephemeris of Smak (1995) was used

the moving averages could be made for the medium level because all phases are covered. Notice the systematic shift of $B-V$ of the high level with respect to the medium and low level. As it will be shown in Fig. 4, $B-V$ displays longterm changes with $\operatorname{mag}(V)$. The series of observations in the intervals of the high level, falling into the same night, are therefore connected by line.

Dependence of the colors on the brightness was examined using $B-V$ and $U-B$ indices, plotted versus $\operatorname{mag}(V)$. Figures 4 and 5 show the color variations for the data within 1995-1997. Resolution of the orbital phases is made, using the different symbols for the phases $0.9-1.1$ (primary eclipse) and the phases 0.1-0.9.

Our data were compared with $U B V$ photometry, published by HPSP. It gives us an opportunity to search for possible long-term color changes. HPSP listed only the out-eclipse observations (phases 0.1-0.9). Their data are plotted as the large squares in the color diagrams (Figs. 4-6).

Histogram of brightness in the $V$ band confirmed that the data tend to cluster in three regions, centered on 10.7, 11.6 and $12.1 \operatorname{mag}(V)$. This separation reflects the fact 
Table 2. $U B V$ photometric observations of V Sge in 1995-1997. The orbital phases and epochs were calculated according to the quadratic ephemeris of Smak (1995). This table in its entirety is available from CDS

\begin{tabular}{ccccccccccr}
\hline JD(hel.) & $V$ & err. & $B$ & err. & $U$ & err. & Phase & Epoch & $U-B$ & $B-V$ \\
\hline 49926.2841 & 10.738 & 0.02 & 10.708 & 0.01 & & & 0.21522 & 23408 & & -0.030 \\
49926.2892 & 10.727 & 0.02 & 10.721 & 0.01 & & & 0.22600 & 23408 & & -0.006 \\
49926.2922 & 10.708 & 0.02 & 10.709 & 0.01 & & & 0.23139 & 23408 & & 0.001 \\
49928.4536 & 10.770 & 0.01 & 10.749 & 0.01 & 9.825 & 0.01 & 0.43586 & 23412 & -0.924 & -0.021 \\
49928.4582 & 10.783 & 0.01 & 10.745 & 0.01 & 9.815 & 0.01 & 0.44485 & 23412 & -0.930 & -0.038 \\
49934.4197 & 11.942 & 0.02 & 11.953 & 0.02 & 11.129 & 0.02 & 0.03723 & 23424 & -0.824 & 0.011 \\
49935.4789 & 12.132 & 0.02 & 12.210 & 0.02 & & & 0.09722 & 23426 & & 0.078 \\
49935.4983 & 11.980 & 0.02 & 12.050 & 0.02 & & & 0.13497 & 23426 & & 0.070 \\
49935.5040 & 12.000 & 0.02 & 12.040 & 0.02 & & & 0.14755 & 23426 & & 0.040 \\
49935.5148 & 11.881 & 0.02 & 11.981 & 0.02 & & & 0.16732 & 23426 & & 0.100 \\
49936.4310 & 12.322 & 0.02 & 12.345 & 0.02 & 11.496 & 0.02 & 0.95049 & 23427 & -0.849 & 0.023 \\
49936.4348 & 12.298 & 0.02 & 12.436 & 0.02 & 11.551 & 0.02 & 0.95768 & 23427 & -0.885 & 0.138 \\
$\ldots$ & $\cdots$ & $\cdots$ & $\cdots$ & $\cdots$ & $\cdots$ & $\cdots$ & $\cdots$ & $\cdots$ & $\cdots$ & $\cdots$ \\
\hline
\end{tabular}

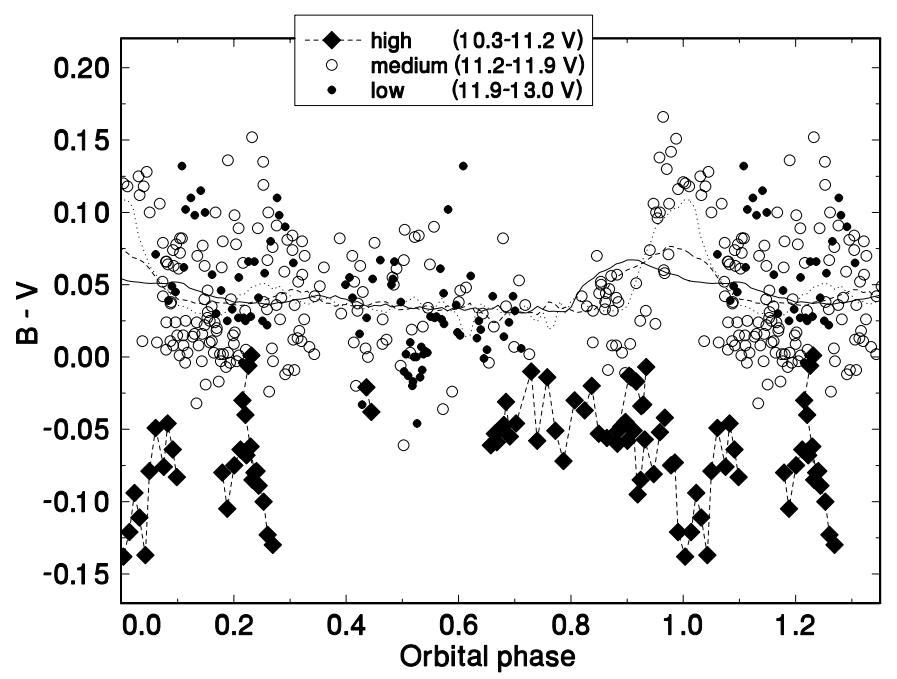

Fig. 3. Dependence of the color index $B-V$ on the orbital phase for various levels of out-eclipse brightness in the $V$-band. The smooth lines represent moving averages of the medium level, calculated for the half-width $Q=0.15$ phase (solid line), $Q=0.10$ (dashed line) and $Q=0.05$ (dot-dashed line), respectively. See text for details

that the long-term brightness variations in $\mathrm{V}$ Sge tend to form relatively discrete levels (see Sect. 4). This clustering complicates the search for the mean course of the color dependence on $\operatorname{mag}(V)$. Moving averages did not yield good fit to the data for 1995-1997. Arithmetic means for the bins of $0.2 \mathrm{mag}(V)$ gave better results. Means for both the whole set and just for the data within phases 0.1-0.9 were made. This division enabled a better comparison with HPSP's out-eclipse data. Another method we applied was the code HEC13 (author Dr. P. Harmanec). This program is based on the method of Vondrák (1969 and 1977) and can fit a smooth curve to the data no matter what their course is. After several trials it was found that the input parameters of the fit $\epsilon=10^{3}$ and the length of the bin $\Delta \mathrm{mag}=0.01$ satisfy the course of the color dependence for both $U-B$ and $B-V$. The input $\epsilon$ parameter determines how "tight" the fit will be (if just the main course or if also the high-frequency variations are to be reproduced). Although the scatter in $B-V$ and $U-B$ is likely to be partly intrinsic because it is larger than the typical observational errors, we concentrated on determination of the overall track in the color diagrams. $\epsilon$ was therefore chosen so that the fit reproduces just the main course. It emerged from the trials that the course of the fitting curve is largely insensitive to $\Delta$ mag for a given $\epsilon$. The number of observations for the brightness lower than about $12.5 \mathrm{mag}(V)$ is small, therefore the fits below this level were truncated. The fit by HEC13 in Fig. 4 shows that the mean $B-V$ index decreases significantly only when the brightness is about $11 \operatorname{mag}(V)$ or brighter. The variation of $B-V$ has a smaller slope below this level. This behaviour is also illustrated by the separate linear fits in Fig. 4. There is no prominent trend in the variations of $U-B$ index over the whole transition (Fig. 5), at most a very small reddening towards higher brightness may be present. The slight curvature of the HEC13 fit in Fig. 5 is likely to be just a result of using the same $\epsilon$ and $\Delta$ mag for both Figs. 4 and 5 .

In Figs. 4 and 5 we also display separately the observations of an almost monotonic rise of brightness which occurred in August 1997 (see also Fig. 1).

Figure 6 shows the $U-B, B-V$ diagram. Both the new data and HPSP's observations are displayed. The new data form a region which is elongated slightly more in the $B-V$ than in $U-B$ direction. HPSP's data basically follow the same trend but the range over which their $B-V$ varies is smaller by about a factor of two. The colors of the main-sequence stars, supergiants, black body radiation along with steady-state disks, modeled by Mayo et al. (1980), are superposed for comparison. Various authors largely differ in the reddening of V Sge. Koch et al. (1986) gives a rather low $E(B-V)=0.2$ while HPSP and Patterson et al. (1998) concluded that $E(B-V)$ is substantially larger and amounts to 0.4 and 0.33 , respectively. Due to these discrepancies we decided to plot the observed data in Fig. 6 and illustrate the dereddening by three vectors, corresponding to the respective values of $E(B-V)$; 


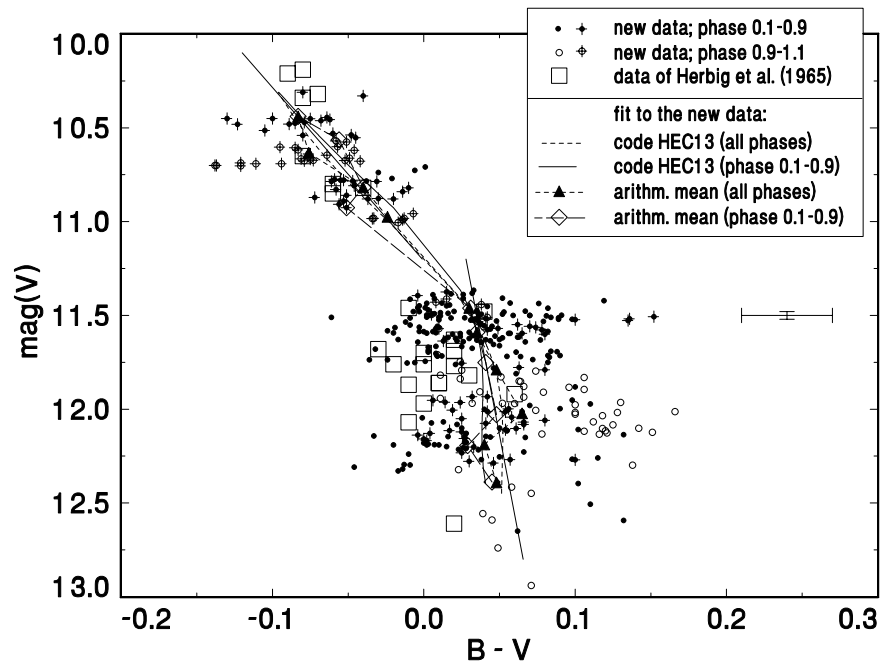

Fig. 4. Color variations $(B-V)$ versus brightness in the $V$ band. The new data represent the years 1995-1997. Resolution of the orbital phases is made - the primary eclipse occurs at phase 0.0. Only the new data were fitted. The thin solid lines represent the linear fits. The crossed symbols denote the observations of an almost monotonic rise of brightness in August 1997. The error bars denote the typical $2 \sigma$ errors of $\operatorname{mag}(V)$ and $B-V$. See text for details

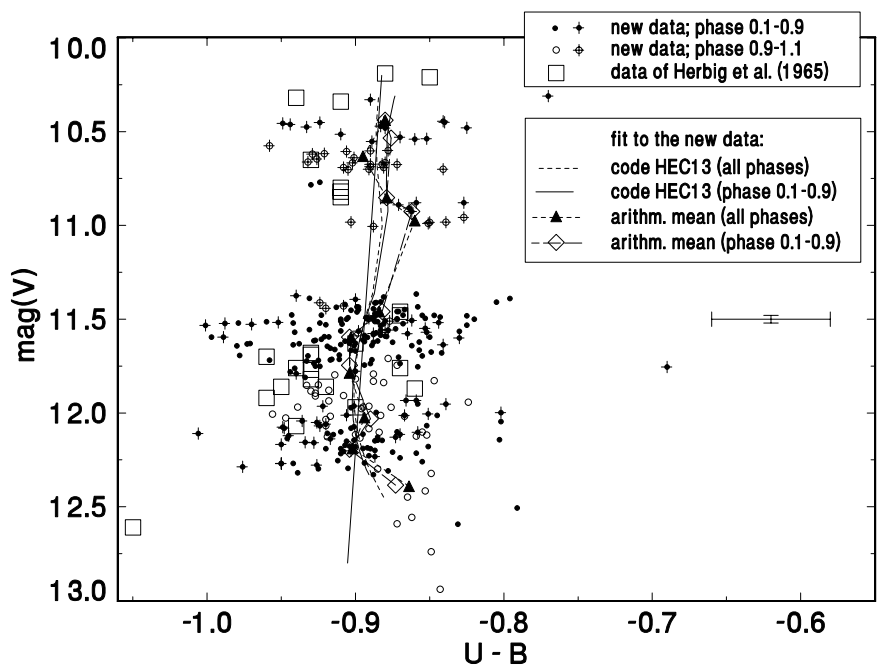

Fig. 5. Color variations $(U-B)$ versus brightness in the $V$ band. The arrangement is the same as in Fig. 4. The error bars denote the typical $2 \sigma$ errors of $\operatorname{mag}(V)$ and $U-B$

each of them denotes the amount by which each observed point should be shifted.

\section{Discussion}

We have presented the color variations of V Sge, representative for its activity over the years 1995-1997. Our observations fall into an active segment (following the notation of Paper I), that is a season in which the brightness varies by about 1.5 to $2 \mathrm{mag}$ and the light curve has a form of the high (HS) and low (LS) states, alternating on the time scale of tens to hundreds of days with relatively rapid transitions. Indeed, the levels of brightness in Fig. 1 are usually typical for HS and LS, analyzed in

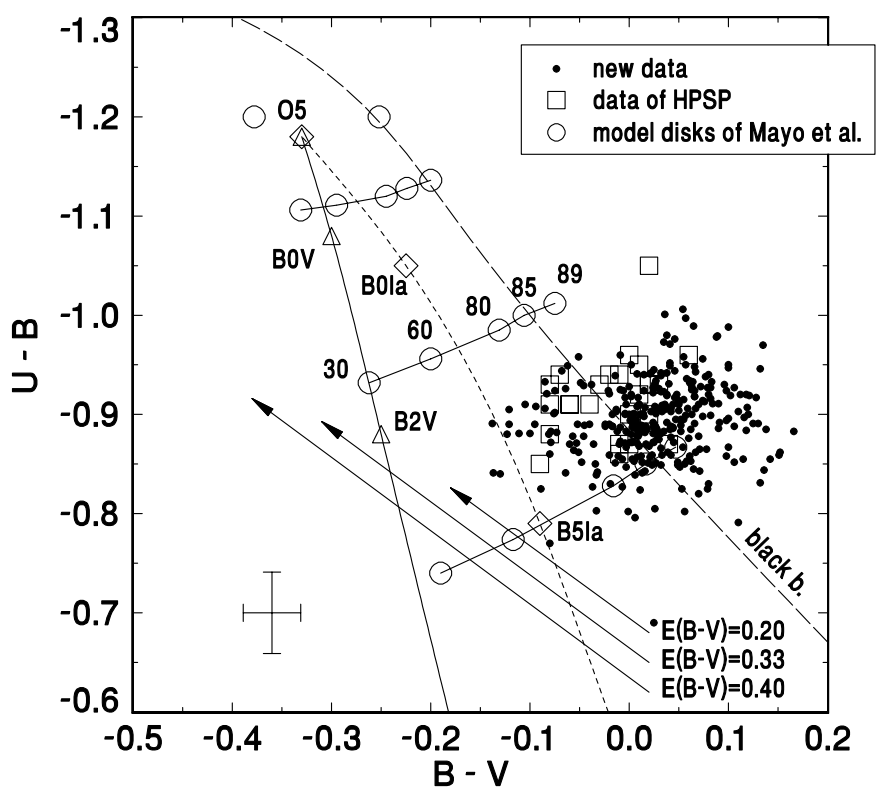

Fig. 6. Color variations in $U-B, B-V$ diagram. Again, the new data represent the years 1995-1997. Their typical $2 \sigma$ errors are marked. The observations of HPSP are plotted for comparison. The arrows represent the reddening given by various authors and mark the amount by which each point should be shifted for the respective values of $E(B-V)$ (HPSP: $E(B-V)=0.4$; Koch et al. 1986: $E(B-V)=0.2$; Patterson et al. 1998: $E(B-V)=0.33)$. Each arrow begins on the mean observed value of $B-V$ of the new data which amounts to 0.02. The colors of the black body radiation (long-dashed line), the main sequence (triangles and solid line), supergiants (inverted triangles and short-dashed line) and the steady-state disks (circles connected by thin solid lines), modeled by Mayo et al. (1980), are superposed. The numbers denote the inclination angles of the theoretical disks in degrees. See text for details

Paper I. However, the level of brightness in the medium state, centered on JD $=2450050$, was roughly intermediate to HS and LS (see also Robertson et al. 1997 and Fig. 4 in Paper I). Moreover, this medium state finished by another decrease into real LS (Fig. 1). All these facts are typical for the previous flat segments, analyzed in Paper I. One may therefore infer that this episode of medium state can possibly be classified as the flat segment, too, although its duration is shorter in comparison with other such intervals (Paper I).

We were able to resolve the tracks of $\mathrm{V}$ Sge in the color diagrams $(B-V$ and $U-B$ versus $\operatorname{mag}(V))$. They cover the full range of brightness between HS and LS, including transition between them. We have shown that the dominant part of the color variations in V Sge must be ascribed to the long-term activity and not to the orbital motion (Figs. 2a-d, 4 and 5). The phase plot of $B-V$ (Fig. 3) is more populated than that in Lockley et al. (1999) and covers the whole range of the brightness variations. Smooth medium level shows an almost constant $B-V$ for most phases and just a small reddening at primary eclipse. However, we note that most points near 
phase 0.0 come from a single eclipse (Fig. 2a) and that the scatter of $B-V$ near the primary eclipse is large. No reddening near phase 0.0 is observed in the high state.

The character of activity in V Sge has changed significantly during the recent decades (Paper I). Nowadays $\mathrm{V}$ Sge spends more time in the high state than in the low state. Also the levels of HS and LS are usually more separated now. On the other hand, a comparison of our new data with HPSP's observations from the early sixties shows that the colors and their variations with the brightness level remained similar. This fact strengthens the evidence (expressed already in Paper I on the base of the long-term trends in the activity) that the process which gives rise to the large variations of brightness in $\mathrm{V}$ Sge remains the same.

The mean $B-V$ gets bluer during the upper part of the LS/HS transition (brighter than approx. $11.3 \mathrm{mag}(V)$ ) The variation of $B-V$ has a smaller slope below this level. We stress that the part of Fig. 4 below approx. $11.3 \mathrm{mag}(V)$ is combined of two states of activity (low and medium). On the contrary, $U-B$ does not change significantly over the whole transition from LS to HS. The fact that $B-V$ decreases at roughly constant $U-B$ (Figs. 4 and 5) may suggest that Balmer jump undergoes just minor changes over LS/HS transition and the continuum gets hotter towards HS. HPSP argued that the entire color variation could be ascribed to the increase in strength of the emission lines. We cannot test this item for the new data because we have no simultaneous spectroscopic observations. However, if HPSP's interpretation is true and if it is valid also for the new data then the change of the slope, present in Fig. 4, suggests that the emission lines strengthen with respect to the continuum mainly when the system is brighter than about $11.5 \mathrm{mag}(V)$. A small systematic difference $\Delta B-V=0.028$ exists between our and HPSP's data for LS and MS (Fig. 4). We cannot rule out that this is due to transformation of a star with a very peculiar spectrum into standard system.

The colors of $\mathrm{V}$ Sge are so blue that $B-V$ and $U-B$, dereddened by the vectors in Fig. 6 , place it to the region where there are just very small differences between the colors of the respective physical objects (black body, stars, disks). One therefore cannot distinguish between changes in the disk, the white dwarf or the donor star just from the $U B V$ colors. The colors of $\mathrm{V}$ Sge correspond to or reach behind the upper end of the distribution of ordinary cataclysmic variables (compare with Bruch 1984). Although one has to be very cautious in comparing the dereddened colors of V Sge with the classic steady-state disks, they do follow an extension of colors of these disks (Mayo et al. 1980) towards higher mass transfer rate (Fig. 6). Some points remain above the black-body line even after dereddening. This fact might be due to Balmer jump in emission, as is the case in some cataclysmic variables (Bruch 1984).

Decrease of $B-V$ mainly in the upper part of the track in Fig. 4 coincides with both the changes of the X-ray spectrum from soft to hard (Greiner \& van Teeseling 1998) and with decrease of the amplitude of the orbital modulation in HS (HPSP, Patterson et al. 1998), attributed to a circumbinary envelope. A radiatively driven wind from the luminous accretion disk (Hachiya et al. 1998) may be the source of this envelope. The smaller $B-V$ changes in the bottom part of the track in Fig. 4 can be also partly ascribed to variations of the dimensions of the disk. Changes of the size of the primary in and near the low state were indeed reported by HPSP.

Acknowledgements. This research has made use of NASA's Astrophysics Data System Abstract Service and partly of the AFOEV database (operated at CDS, France). We are indebted to Dr. Harmanec for providing us with the program HEC 13. We thank Dr. Hudec for reading the manuscript and for his comments. The research of V.S. is supported by the postdoctoral grant 205/00/P013 of the Grant Agency of the Czech Republic. Investigation of cataclysmic variables is partly supported by the project KONTAKT ME 137 by the Ministry of Education and Youth of the Czech Republic. This study was also supported in part by the Russian Foundation for Basic Research and the Council of the Program for the "Support of Leading Scientific Schools" through grants No. 99-02-17589 and 96-15-96489.

\section{References}

Bruch, A. 1984, A\&AS, 56, 441

Eracleous, M., Halpern, J., \& Patterson, J. 1991, ApJ, 382, 290

Greiner, J., \& van Teeseling, A. 1998, A\&A, 339, L21

Hachiya, M., Tajima, Y., \& Fukue, J. 1998, PASJ, 50, 367

Herbig, G. H., Preston, G. W., Smak, J., \& Paczynski, B. 1965, ApJ, 141, 617 (HPSP)

Hoard, D. W., Wallerstein, G., \& Willson, L. A. 1996, PASP, 108, 81

Koch, R. H., Corcoran, M. F., \& Holenstein, B. D. 1986, ApJ, 306,618

Lockley, J. J., Eyres, S. P. S., \& Wood, J. H. 1997, MNRAS, 287, L14

Lockley, J. J., Wood, J. H., Eyres, S. P. S., Naylor, T., \& Shugarov, S. 1999, MNRAS, 310, 963

Mayo, S. K., Wickramasinghe, D. T., \& Whelan, J. A. J. 1980, MNRAS, 193, 793

Meyer-Hofmeister, E., Schandl, S., \& Meyer, F. 1997, A\&A, 321,245

Patterson, J., Kemp, J., Shambrook, A., et al. 1998, PASP, 110,380

Robertson, J. W., Honeycutt, R. K., \& Pier, J. R., 1997, AJ, 113,787

Schandl, S., Meyer-Hofmeister, E., \& Meyer, F. 1997, A\&A, 318,73

Smak, J. 1995, Acta Astron., 45, 361

Steiner, J. E., \& Diaz, M. P. 1998, PASP, 110, 276

Šimon, V., \& Mattei, J. A. 1999, A\&AS, 139, 75, Paper I

van den Heuvel, E. P. J., Bhattacharya, D., Nomoto, K., \& Rappaport, S. A. 1992, A\&A, 262, 97

Vondrák, J. 1969, Bull. Astron. Inst. Czechosl., 20, 349

Vondrák, J. 1977, Bull. Astron. Inst. Czechosl., 28, 84

Williams, G. A., King, A. R., Uomoto, A. K., \& Hiltner, W. A. 1986, MNRAS, 219, 809 